\title{
Type of dike using C-shaped vertical cylinders
}

\author{
Guillaume Dupont, ${ }^{1,{ }^{*}}$ Fabien Remy, ${ }^{1}$ Olivier Kimmoun, ${ }^{1}$ Bernard Molin, ${ }^{1}$ Sebastien Guenneau, ${ }^{2}$ and Stefan Enoch ${ }^{2}$ \\ ${ }^{1}$ Aix Marseille Univ, CNRS, Centrale Marseille, IRPHE UMR 7342, 13013 Marseille, France \\ ${ }^{2}$ Aix Marseille Univ, CNRS, Centrale Marseille, Institut Fresnel UMR 7249, 13013 Marseille, France
}

(Received 16 March 2017; published 15 November 2017)

\begin{abstract}
The present study investigates a way to design dikes which can filter the wavelengths of ocean surface waves. This offers the possibility to achieve a structure that can attenuate waves associated with storm swell, without affecting coastline in other conditions. Our approach is based on low-frequency resonances in metamaterials combined with Bragg frequencies for which waves cannot propagate in periodic lattices.
\end{abstract}

DOI: 10.1103/PhysRevB.96.180302

Over the last decade, major natural disasters due to large storms have impacted coastal zones, leading to important flooding with material and human losses. The water levels associated with storms, at coastal locations, are a combination of storm surges, local tides, and storm waves.

In contrast with the storm surge and the tide which are long waves (several hours), the storm swell consists of short waves $(10-20 \mathrm{~s})$ and can be characterized by large significant wave heights and, in most cases, an increase of their periods with respect to the case without a storm. Those waves can contribute to the surge by transferring their energy when breaking, and, they can impact structure inundated by the surge which can be extremely destructive. Moreover, even with a small surge event, short waves can be severe and significant leading to a strong impact on the coastline with possibly the occurrence of overflow [1].

Under those observations, we propose a way to consequently reduce the contribution of storm swell, in order to protect coastal zones.

Our study is built upon the works on periodic structures in the area of metamaterials and more specifically on lowfrequency stop bands and band gaps [2].

In the context of metamaterials for water waves, some works have been done in recent years in order to render objects invisible to waves. This cloaking approach has allowed one to propose some developments to protect structures or areas in the open sea [3-10] for most of the cases, but also to control the trajectories of waves. Regarding the control of water wave trajectories, periodic structures present some interesting properties. The study of such structures in solid-state physics and optics has emerged in the area of photonic crystals [2], and has been translated in water wave physics [11-17].

In our study, we propose to use a periodic array of C-shaped cylinders (split tubes), also known as split-ring resonators for artificial magnetism in optics [18]. Such a lattice has already been used to observe negative effective gravity [19]. However, another important feature of this kind of lattice is to have a low-frequency band gap and low-frequency stop band [20], at variance with plain cylinder arrays. Thus, it is possible to define a periodic array with constituents of reasonable size with regard to wavelength that can attenuate waves associated with storm swell (where the period is increased relative to mild

*Corresponding author: dupont.guillaume@ centrale-marseille.fr sea state) but not the waves associated with normal sea state. This means that the coastline is not impacted by the presence of the structure in the case of normal sea state but that the structure is "active" only when the wave period is long.

In this Rapid Communication, we demonstrate numerically and experimentally in the case of linear water wave theory the efficiency of a periodic lattice composed of C-shaped cylinders to protect coastal zones. The numerical study is used as a starting point to give directions and it is sufficient to emphasize the general behavior of the structure for linear waves, so we do not reflect the dissipative contribution. In addition, we show that such a structure remains efficient when the amplitude of waves increases (i.e., when the nonlinearities become nonnegligible).

We consider a fluid domain of water depth $h$ with an infinite periodic lattice of rigid, vertical and bottom mounted objects. The Cartesian coordinate system is chosen with $x$ and $y$ as horizontal directions and $z$ the vertical upward direction with origin taken at the mean free surface. We assume linearized potential flow theory of water waves, where the fluid is taken as inviscid, incompressible with irrotational flow, to describe the propagation of water waves with angular frequency $\omega$ through the array. Under these assumptions, the velocity potential can be written as

$$
\Phi(x, y, z, t)=\operatorname{Re}\left\{\phi(x, y) \frac{\cosh (k(z+h))}{\cosh (k h)} e^{-i \omega t}\right\} .
$$

$\phi$ satisfies the Helmholtz equation

$$
\left(\nabla^{2}+k^{2}\right) \phi=0
$$

in the fluid domain, with $\nabla=(\partial / \partial x, \partial / \partial y)^{T}$ and the wave number $k$ solution of the dispersion equation

$$
\omega^{2}=g k \tanh (k h)
$$

with $g$ the gravitational acceleration.

In addition, Neumann boundary conditions

$$
\nabla \phi \cdot n=0
$$

are assumed on the rigid object surfaces, where $\boldsymbol{n}$ is outward unit normal vector.

The lattice consists of an array of C-shaped cylinders defined by the two lattice vectors $\boldsymbol{a}_{1}$ and $\boldsymbol{a}_{\mathbf{2}}$ [see Fig. 1(a)] so that it can be represented by all the translations $\boldsymbol{R}$ of a 
(a)

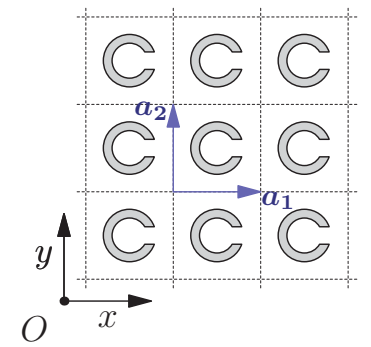

(b)

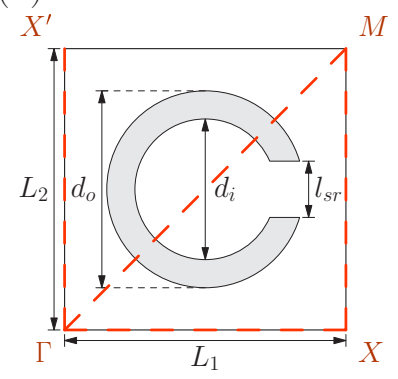

FIG. 1. (a) Top view of a two-dimensional rectangular lattice with lattice vectors $\boldsymbol{a}_{1}$ and $\boldsymbol{a}_{2}$, composed of C-shaped cylinders. (b) Periodic cell of the lattice spanned by $\boldsymbol{a}_{1}$ and $\boldsymbol{a}_{2}$, with $d_{o}$ the outer diameter, $d_{i}$ the inner diameter, and $l_{s r}$ the width of the slit of cylinders. The points $\Gamma X M X^{\prime}$ denote the extrema of the irreducible Brillouin zone and the dashed lines denote directions that bound this zone.

periodic pattern displayed in Fig. 1(b), with

$$
\boldsymbol{R}=n_{1} \boldsymbol{a}_{\mathbf{1}}+n_{2} \boldsymbol{a}_{2},
$$

where $n_{1}$ and $n_{2}$ are integers.

Following the theory used in the study of crystal structures in solid-state physics [21] and in the photonic crystals area [2], we look for solutions of problem (2) using Bloch waves decomposition. In this context, a solution $\phi$ of (2) can be written as the product of a plane wave with a function $\psi$ which has the periodicity of the lattice:

$$
\phi(\boldsymbol{r})=e^{i \boldsymbol{q} \cdot \boldsymbol{r}} \psi(\boldsymbol{r}) \quad \text { with } \quad \psi(\boldsymbol{r})=\psi(\boldsymbol{r}+\boldsymbol{R}),
$$

which is equivalent to

$$
\phi(\boldsymbol{r}+\boldsymbol{R})=e^{i \boldsymbol{q} \cdot \boldsymbol{R}} \phi(\boldsymbol{r}),
$$

where $\boldsymbol{q}$ is the Bloch (real-valued) vector defined in the Brillouin zone.

In our case, the Brillouin zone defines a rectangular cell in reciprocal space with $q \in\left[-\pi / L_{1} ; \pi / L_{1}\right] \times$ $\left[-\pi / L_{2} ; \pi / L_{2}\right]$, which can be further reduced to the irreducible Brillouin zone with $\boldsymbol{q} \in\left[0 ; \pi / L_{1}\right] \times\left[0 ; \pi / L_{2}\right]$ [see Fig. 1(b)].

Solving the eigenvalue problem (2) with the condition (7) amounts to defining values of $\boldsymbol{q}$ irreducible Brillouin zone and solving for all $\boldsymbol{q}$. This method leads to nontrivial eigensolutions $\phi$ for any pair $(k, \boldsymbol{q})$, which are plotted on a band diagram.

In the present study we consider square lattices with $L_{1}=L_{2}=L$, composed of rigid vertical and bottom mounted cylinders with $C$ shape [see Figs. 1(a) and 1(b)]. We study three different configurations where geometric parameters are reported in Table I, with $H / \lambda$ the waves steepness $(H / \lambda=2 a / \lambda$ with $a$ and $\lambda$, respectively, the amplitude and

TABLE I. Set of geometric parameters corresponding to the studied configurations.

\begin{tabular}{lccccc}
\hline \hline & $L(\mathrm{~m})$ & $d_{i}(\mathrm{~m})$ & $d_{o}(\mathrm{~m})$ & $l_{s r}(\mathrm{~m})$ & $H / \lambda(\%)$ \\
\hline cfg. 1 & 0.325 & 0.144 & 0.150 & 0.025 & $2 ; 5$ \\
cfg. 2 & 0.650 & 0.290 & 0.300 & 0.050 & 2 \\
cfg. 3 & 0.650 & 0.290 & 0.300 & 0.160 & $2 ; 3.5$ \\
\hline \hline
\end{tabular}

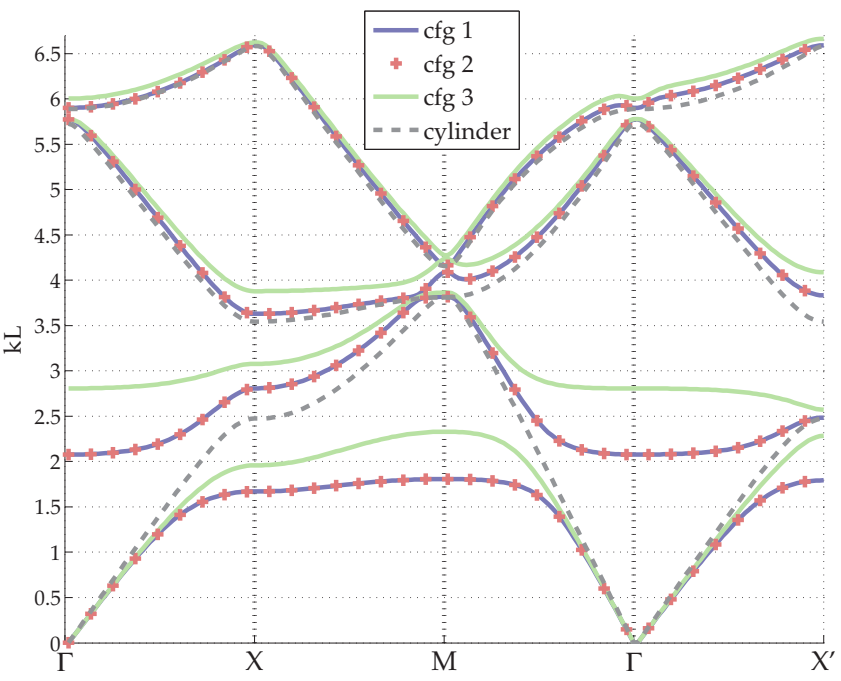

FIG. 2. Band diagrams corresponding to the configurations mentionned in Table I. The dashed lines stand for bands associated with a square lattice $L=0.325 \mathrm{~m}$ of plain cylinders with diameter $d_{o}=0.150 \mathrm{~m}$.

the wavelength of incoming waves). The configuration 2 is quite close to the configuration 1 where all the parameters have been multiplied by a factor $\sim 2$. This will illustrate that the approach is valid from deep to shallow water. We note that the only difference between configurations 2 and 3 is the size of the slit $l_{s r}$.

As a first step, the eigenvalue problem given by Eqs. (2) and (4) is solved using a finite element (FE) Galerkin method, for Bloch waves, for each of the configurations. The results are shown in Fig. 2, according to the normalized wave number $k L$. We first notice the quasi-identical results for configurations 1 and 2, which means that a scale factor on the lattice is passed on the wave number, that is, on the wavelength. For all cases, a band gap is observed between the first two bands corresponding to low frequencies, which is due to the presence of the slit (note that an extra path along $\Gamma X^{\prime}$ is required in the band diagram [22]). This behavior is not observed in the case of plain cylinders (see Fig. 2, dashed curves). For the inner and outer diameters we choose, we note the larger the slit (configuration 3), the larger the first stop band and the thinner the second one.

The $\Gamma X$ part of the diagram corresponds to waves propagating along principal directions $\left(\boldsymbol{a}_{1}, \boldsymbol{a}_{2}\right)$ in the array. It appears on this part, two significant partial stop bands, respectively, between the first and second bands and between the second and third bands. It means that waves with frequencies in those stop bands are disallowed to propagate in the array along principal directions. Or alternatively, waves with frequencies in those stop bands coming from free space normally to such a periodic array are reflected and do not propagate through the array. Moreover, for some frequencies in the first stop band, waves do not propagate through the array for all angles of incidence.

The previous arguments suggest that a periodic array with $\mathrm{C}$-shaped cylinders can be used as a dike that is capable of stopping waves over a significant range of wavelengths and 


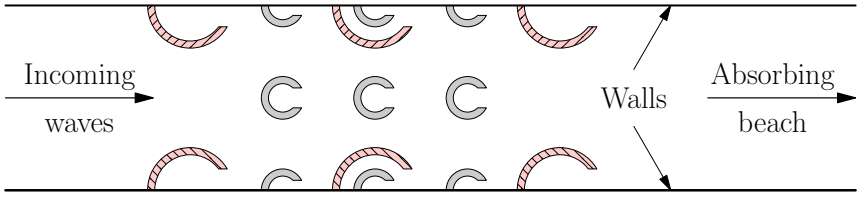

FIG. 3. Schematic top view of the experimentally tested arrays. Array with smaller size C-shape cylinder (gray) corresponding to configuration 1 in Table I. Array with larger size C-shape cylinder (red with hatch) corresponding to configurations 2 and 3.

with even more efficiency for waves propagating with small angles around a principal direction.

To illustrate the purpose, we numerically investigate the interaction of regular waves with the arrays whose parameters are given in Table I. We consider a numerical wave tank with constant water depth $h=0.5 \mathrm{~m}$ where lattices organized as in Fig. 3 take place. Those choices of organization for our lattices are motivated by our experimental setup. For configuration 1, we have $k h \in[1.5 ; 9.3]$ which corresponds to water waves in intermediate and deep water and we have $k h \in[0.7 ; 4.7]$ for configurations 2 and 3 , which corresponds to water waves in intermediate water, close to shallow water for smaller $k h$. Incoming waves are at normal incidence, which corresponds to waves propagating in the $\Gamma-X$ direction.

We use the Helmholtz equation [Eq. (2)] combined with a FE Galerkin method to calculate the free-surface elevation.

Figure 4 shows the transmission coefficients with respect to the normalized wave number $k L$ for our three configurations. As predicted by the band diagrams, results are identical for configurations 1 and 2 . We easily identify the band gaps at small $k L$ (low frequencies) for which the transmission coefficient is null, and the stop bands at larger $k L$ for which the

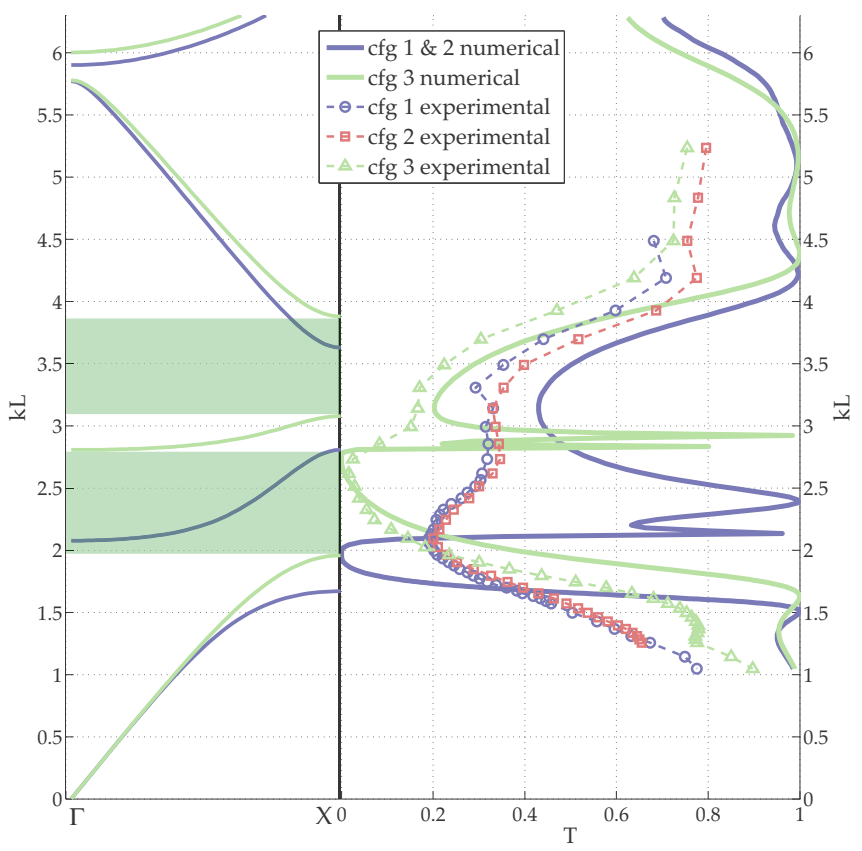

FIG. 4. Comparison of experimental measurements of the transmision coefficient with numerical results for incident water waves along $\Gamma X$ with wave steepness $H / \lambda=2 \%$.

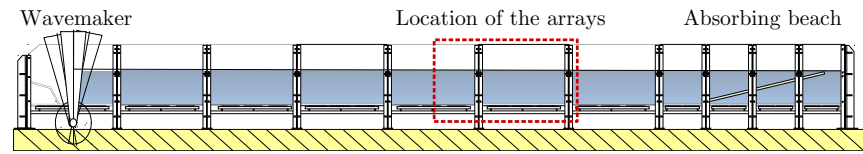

FIG. 5. Schematic side view of the wave tank located at engineering school Centrale Marseille.

transmission coefficient is low. Those two gaps are separated by a peak of transmission corresponding to the second band.

For the experimental part of the study, we use the wave tank at engineering school Centrale Marseille, which is $17 \mathrm{~m}$ long and $0.65 \mathrm{~m}$ wide. Waves are generated by a flap wave maker, the center of the arrays is located at $10 \mathrm{~m}$ from the wave maker, and an efficient 3-m-long absorbing beach takes place at the end of the tank (see Fig. 5).

The amplitudes of reflected and transmitted waves are measured with sets of resistive wave gauges placed in front and behind the arrays. Additionally, we put a wave gauge inside a cylinder of each column of the array. Each configuration is tested for different wave steepness $(H / \lambda$ entries in Table I).

Figure 4 shows the measured transmission coefficients for incident wave steepness $H / \lambda=2 \%$, compared with numerical results. An important feature is that we did not observe experimentally the peak of transmission corresponding to the second band, for all the configurations. On the other hand, we note similar results for configurations 1 and 2, with a reasonable agreement with numerical computations, in the sense that the global variation is respected. Differences with numerics are presumably due to dissipative phenomena, such as flow separation induced by sharp corners, viscous effects, and wave breaking. Results for configuration 3 , where the size of the slit has been increased, are meanwhile in good agreement with numerical results, especially for the minima.

A part of the explanation for transmission associated with the second band can be found in the measurement of amplitude inside cylinders. The results for configuration 3 displayed in Fig. 6 show that the normalized amplitude becomes important

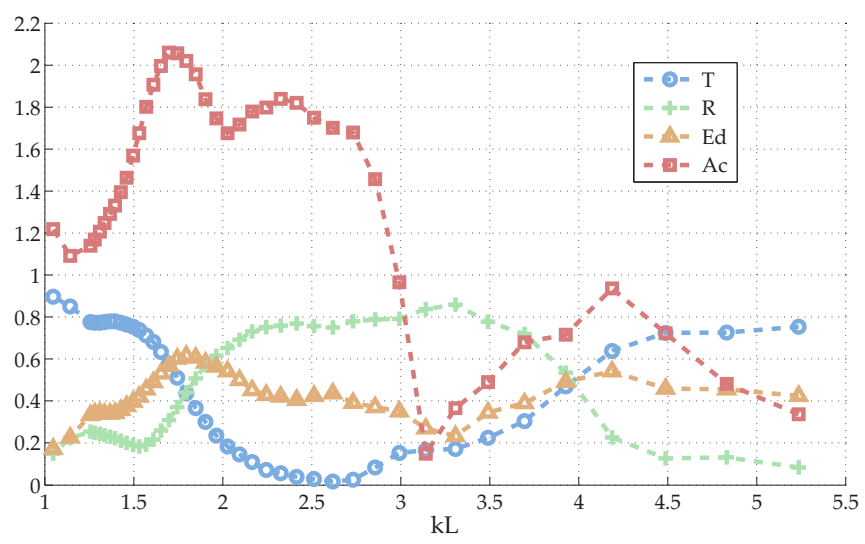

FIG. 6. Experimental measurements of the transmission coefficient $(\mathrm{T})$, reflection coefficient $(\mathrm{R})$, normalized amplitude inside a cylinder of the first column (Ac), and dissipation coefficient (Ed) [see Eq. (8)] for configuration 3. The amplitude inside the cylinders for other columns is similar but attenuated. 


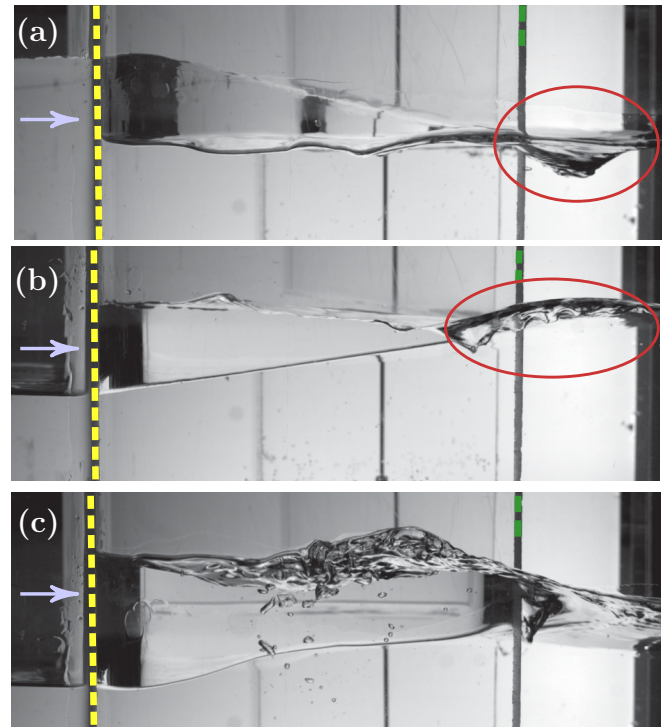

FIG. 7. Photos of a cylinder at different time steps and for different wave steepnesses. Photos are side views where the waves propagate from the left (arrows), the dashed yellow lines are the plain side of the cylinder, and the dashed green lines indicate the side with the split. Cylinders are transparent such that we see water inside and behind. (a) Water comes out of the cylinder for $H / \lambda=2 \%$, (b) water comes in the cylinder for $H / \lambda=2 \%$, and (c) wave breaking in the cylinder for $H / \lambda=5 \%$. The vortices are identified inside the red circles.

inside the cylinder for $k L<3$, which includes the second band. Additionally, for those values of $k L$, an important flow separation, with generation of vortices, is observed on both sides of the slit when water waves come in and out the cylinder [see Figs. 7(a) and 7(b)]. For values of $k L$ where the amplitude is maximal inside the cylinder, wave breaking occurs [see Fig. 7(c)].

The dissipation coefficient

$$
E_{d}=1-\left(R^{2}+T^{2}\right)
$$

which is zero according to linearized potential flow theory, confirms that when the amplitude becomes significant inside cylinders, dissipation increases (yellow curve with triangle markers in Fig. 6). This fact is observed for all the configurations (see Fig. 8). Additionally, a part of the wave energy is transferred to higher harmonic components.

Increasing the amplitude of incident water waves (wave steepness up to $5 \%$ ), we obtain results very similar to waves with small amplitudes, as depicted in Fig. 9. The amplitude of transmitted waves can be attenuated by $50 \%$ for a wide range of wavelengths: $1.5<k L<4$ which corresponds to $4 L>$ $\lambda>1.5 L$, by adjusting the size of the slit. More preferably, the attenuation of transmitted waves reaches $70 \%-80 \%$ for $2<k L<3.5(3.2 L>\lambda>1.8 L)$. A video of a run test for $k l=2.5$ and $H / \lambda=7 \%$ is provided as Supplemental Material [23].

Those observations evidence that this kind of array makes a good choice to consequently minimize the impact of storm swell on coastal zones.

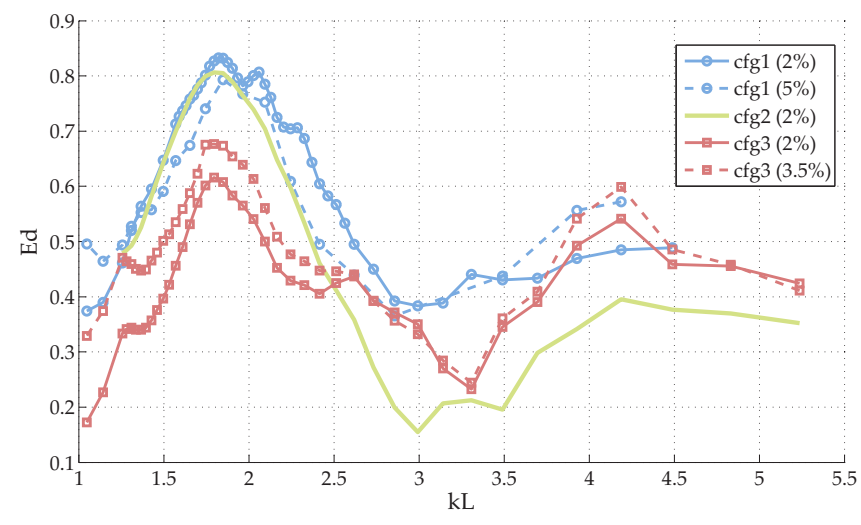

FIG. 8. Dissipation coefficients for all the studied configurations. When the amplitude of waves is important in the cylinder $(k L \lesssim 2.7)$, we note that, the thinner the slit (configurations 1 and 2), the higher the dissipation.

In this Rapid Communication, we have performed numerical and experimental demonstrations about a periodic structure that can be used as a dike to protect coastal zones.

In a first step, starting with the study of an infinite periodic lattice, we prove numerically that our choice of C-shaped cylinders may induce a large low-frequency stop band associated with a low transmission on a wide range of wavelengths. In a second step, we illustrate it experimentally and we extend the study to waves with large wave heights, which confirms the positive action of the structure on storm swell.

We stress that, with the knowledge of local parameters of a coastal zone (bathymetry, periods of waves), it is always possible to define a structure in our way, thanks to the link between wavelengths and the lattice parameter $L$. Then, for an array defined for waves with $k L \sim 5$ (normal sea state, maximum of transmission, invisible structure to water waves), a significant increase of the period of waves will be associated with a low transmission and a consequent attenuation of the amplitude of waves. That means the structure

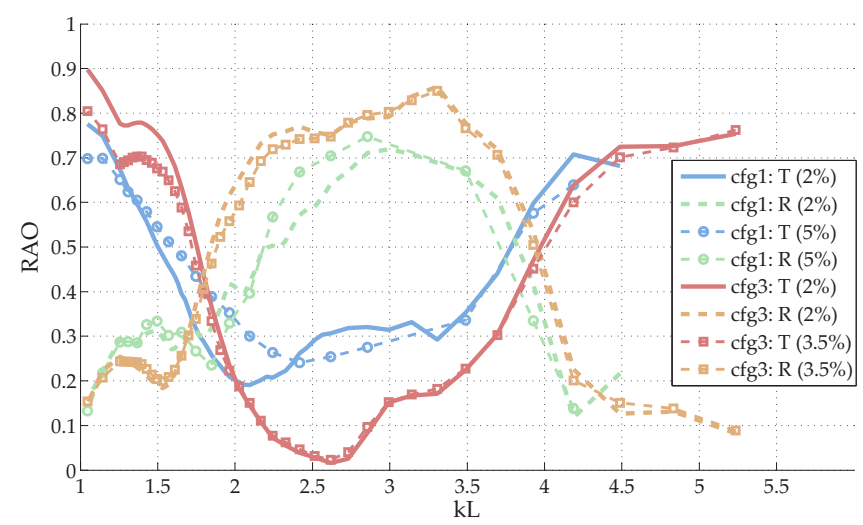

FIG. 9. Comparison of experimental reflection and transmission coefficients for different wave steepnesses (shown in parentheses). Results for higher wave steepnesses are represented with markers (circles for configuration 1 and squares for configuration 3 ). 
becomes active. On the other hand, when the period of waves decreases, the associated band structure flattens and, as for the second band, we should not capture thin peaks of transmission.
Consequently, we are convinced that our results pave the way for a technology of dikes, active for most cases of storm swell. This technology may help to preserve the environment and is less invasive than classical dikes.
[1] National Oceanic and Atmospheric Administration, http://www.noaa.gov/

[2] J. D. Joannopoulos, S. G. Johnson, J. N. Winn, and R. D. Meade, Photonic Crystals: Molding the Flow of Light (Princeton University Press, Princeton, NJ, 2011).

[3] M. Farhat, S. Enoch, S. Guenneau, and A. B. Movchan, Phys. Rev. Lett. 101, 134501 (2008).

[4] M.-R. Alam, Phys. Rev. Lett. 108, 084502 (2012).

[5] J. Newman, European J. Mech. B/Fluids 47, 145 (2014), Enok Palm Memorial Volume.

[6] R. Porter and J. Newman, J. Fluid Mech. 750, 124 (2014).

[7] A. Zareei and M.-R. Alam, J. Fluid Mech. 778, 273 (2015).

[8] G. Dupont, S. Guenneau, O. Kimmoun, B. Molin, and S. Enoch, J. Fluid Mech. 796, 249 (2016).

[9] C. P. Berraquero, A. Maurel, P. Petitjeans, and V. Pagneux, Phys. Rev. E 88, 051002 (2013).

[10] G. Dupont, O. Kimmoun, B. Molin, S. Guenneau, and S. Enoch, Phys. Rev. E 91, 023010 (2015).

[11] C. Linton and P. McIver, J. Eng. Math. 30, 661 (1996).

[12] P. McIver, J. Fluid Mech. 424, 101 (2000).
[13] X. Hu, Y. Shen, X. Liu, R. Fu, and J. Zi, Phys. Rev. E 69, 030201 (2004).

[14] X. Hu and C. T. Chan, Phys. Rev. Lett. 95, 154501 (2005).

[15] M. A. Peter, M. H. Meylan, and C. Linton, J. Fluid Mech. 548, 237 (2006).

[16] M. Farhat, S. Guenneau, S. Enoch, and A. Movchan, J. Comput. Appl. Math. 234, 2011 (2010).

[17] M. McIver, Q. J. Mech. Appl. Math. 67, 631 (2014).

[18] J. B. Pendry, A. J. Holden, D. J. Robbins, and W. J. Stewart, IEEE Trans. Microwave Theory Tech. 47, 2075 (1999).

[19] X. Hu, J. Yang, J. Zi, C. T. Chan, and K.-M. Ho, Sci. Rep. 3, 1916 (2013).

[20] A. B. Movchan and S. Guenneau, Phys. Rev. B 70, 125116 (2004).

[21] C. Kittel, Introduction to Solid State Physics (Wiley, New York, 1966), Vol. 162.

[22] A. Lardeau, J.-P. Groby, and V. Romero-García, Crystals 6, 51 (2016).

[23] See Supplemental Material at http://link.aps.org/supplemental/ 10.1103/PhysRevB.96.180302 for a video of a run test. 